\title{
Crack Localization Using Transmissibility of Operational Deflection Shape and Its Application in Cantilever Beam
}

\author{
X. Z. Li, X. B. Yue, W. Huang \\ Institute of Mechanical Manufacturing Technology, China Academy of Engineering Physics, Mianyang, China \\ Email: li_xingzhan@126.com
}

How to cite this paper: Li, X.Z., Yue, X.B. and Huang, W. (2018) Crack Localization Using Transmissibility of Operational Deflection Shape and Its Application in Cantilever Beam. Journal of Applied Mathematics and Physics, 6, 2352-2361. https://doi.org/10.4236/jamp.2018.611197

Received: November 5, 2018 Accepted: November 19, 2018 Published: November 22, 2018

\begin{abstract}
Due to the nonlinearity of breathing crack, cracked structure under excitation of a single frequency always generates higher harmonic components. In this paper, operational deflection shape (ODS) at excitation frequency and its higher harmonic components are used to map the deflection pattern of cracked structure. While ODS is sensitive to local variation of structure in nature, a new concept named transmissibility of operational deflection shape (TODS) has been defined for crack localization using beam-like structure. The transmissibility indicates the energy transfer from basic frequency to higher frequency. Then, Teager energy operator (TEO) is employed as a singularity detector to reveal and characterize the features of TODS. Numerical and experimental analysis in cantilever beam show that TODS has strong sensitivity to crack and can locate the crack correctly.
\end{abstract}

\section{Keywords}

Crack Localization, Breathing Crack, Operational Deflection Shape, Transmissibility

\section{Introduction}

Detection and location of cracks at an early stage of their development is an important part of structural health monitoring system for protecting the structure from serious damage. Over the past few decades, vibration based methods have widely been used in crack detection for different types of structures [1]. Among all these methods, one kind of method using operational deflection shape (ODS) draw great attention in damage detection. Ratcliffe [2] proposed the gapped smoothing method (GSM) to deal with the broadband ODS, where the unda- 
maged beam structures are not needed if they are geometrically smooth and made of materials that have no stiffness and mass discontinuities. The damage index was shown as a contour plot of frequency versus position. Yoon et al. [3] expanded the GSM and developed a Global Fitting Method by introducing a globally optimized smooth shape using ODS to improve the performance of damage detection for various damage. Zhang et al. [4] proposed the Global Filtering Method based on ODS curvature extracted from dynamic response of a passing vehicle. Only ODS at few frequencies near the first natural frequency is needed and pre-filtering process is applied on the ODS to reduce the numerical error. A vibration testing method using superposed waveform method (SWM) is proposed by Feng and Li [5] for damage detection in structures. Propagating wave data were acquired by a scanning laser Doppler vibrometer to calculate the ODSs at each frequency using the SWM.

Since it is not always possible to locate cracks in structures only using the ODS [6], a method combining the ODS with transmissibility is proposed in this study. Zhang et al. [7] and Mottershead [8] demonstrated that transmissibility is determined solely by system zeros, which makes the transmissibility sensitive to the local variation in nature. ODSs provide the geometric feature of structure, while conventional transmissibility shows the energy transfer from point to point. Under the excitation of a single frequency, structure with nonlinear breathing crack will generate higher harmonic components, which contain the information of damage location. A new concept named transmissibility of operational deflection shape (TODS) has been defined in present paper. The new transmissibility is estimated using the ratio between the higher harmonic ODS and basic harmonic ODS, which shows the energy flow. Energy transformation in the crack position will be discontinuous. Teager energy operator (TEO) is employed as a singularity detector to reveal this feature of TODS.

The paper is organized as follows. Section 2 presents the definition of TODS based on the conventional transmissibility, and the procedure for crack location using the dynamic response. Section 3 shows several numerical simulations to verify the effectiveness of indicator. Section 4 describes the experiment used to validate the new method in cantilever beam. Finally, conclusions are presented in Section 5.

\section{Transmissibility of Operational Deflection Shape and the Procedure for Crack Location}

\subsection{Recap of Conventional Transmissibility}

Contrary to the popular frequency response functions (FRF) which are frequency relationship between dynamic response and force input, transmissibility is defined as the ratio of two dynamic response spectra. Assuming a single force is applied in the input degree of freedom (DOF), it is verified that the transmissibility can be expressed as 


$$
T_{i j}(\omega)=\frac{X_{i}(\omega)}{X_{j}(\omega)}=\frac{H_{i k}(\omega) F_{k}(\omega)}{H_{j k}(\omega) F_{k}(\omega)}=\frac{N_{i k}(\omega)}{N_{j k}(\omega)}
$$

where $H_{i k}(\omega)$ and $H_{j k}(\omega)$ are the transfer functions. $N_{i k}(\omega)$ and $N_{j k}(\omega)$ are the numerator polynomials corresponding to the different outputs. $X_{i}(\omega)$ and $X_{j}(\omega)$ are the response spectra. $F_{k}(\omega)$ is the input spectrum. It can be observed that the common denominator, whose roots are the system's poles, vanishes by taking the ratio of the two response spectra. Consequently, the poles of the transmissibility equal the zeroes of transfer function. In general, the peaks in the amplitude of transmissibility do not coincide with the resonances of the system. From the Equation (1), one important property of transmissibility can be derived. Transmissibility is independent to the input and system poles, but is dependent on the location of input and system zeros. While system poles is function of all the system dynamic parameters, system zeros is influenced by the local subset. Hence, transmissibility is sensitive to the local variation in nature, which gives the advantage for damage detection.

\subsection{Transmissibility of Operational Deflection Shape}

A breathing crack opens and closes alternatively during every cycle of loading and consequently produces the nonlinear phenomenon of the cracked structure. When the cracked structure is excited at a single frequency, higher harmonics of the exciting frequency are generated due to the nonlinear dynamic. Since the nonlinearity is generated by the crack, higher harmonics can be used to locate the crack. The ODS of the cracked beam can be generated at the exciting frequency and the higher harmonic frequencies to map the deflection of the cracked structure. However, it has been observed from [6] that the ODSs at the higher harmonics cannot always distinguish the crack location due to the influence by the ODS at the frequency of excitation. As a result, in order to reduce the effect of the basic harmonic and the nonlinearity due to the breathing crack, transmissibility of the ODSs (TODSs) between the higher harmonics ODSs and the basic harmonic ODS are proposed in this study.

$$
T O D S_{m, 1}=\frac{O D S_{k, m}}{O D S_{k, 1}}
$$

where $O D S_{k, m}$ is the m-th harmonic ODS at mode k. TODS $S_{m, 1}$ is the transmissibility of ODS between $\mathrm{m}$-th harmonic and basic harmonic. Since the transmissibility represents the energy transfer, TODS shows the energy transfer from the basic harmonic ODS to the higher harmonic ODSs. The energy transfer before and after the crack will changes since vibration energy dissipates to thermal energy with the open and close of crack, which can be used to locate the nonlinearity induced by breathing crack.

\subsection{Teager Energy Operator of TODS}

The energy transfer will generate the singular phenomenon. To detect and high- 
light this singularity, a popular operator is applied in the procedure of crack location. The discrete Teager energy operator (TEO) was proposed with the aim of representing the transient energy of a signal. For a discrete sequence $f[n]$, the TEO is defined as

$$
\Psi(f[n])=f^{2}[n]-f[n-1] f[n+1]
$$

where $\Psi(f[n])$ denotes the TEO that calculates the approximate transient energy of $f[n]$. The transient property makes the TEO an effective nonlinear operator to locate the singularity of a signal, which is suitable for the analysis of global characteristics. As a result, the TEO in this study is adopted to character the abnormality of the TODS that is caused by local breathing crack. Implementation of the TEO on TODS is expressed as

$$
\operatorname{TTODS}_{m, 1}[n]=\left(\operatorname{TODS}_{m, 1}[n]\right)^{2}-\operatorname{TODS}_{m, 1}[n-1] * \operatorname{TODS}_{m, 1}[n+1]
$$

where $\operatorname{TTODS}_{m, 1}[n]$ is defined as the Teager Energy Operator of TODS (TEO-TODS) between the $\mathrm{m}$-th harmonic ODS and basic harmonic ODS, which is used as a new indicator for damage location in beam-like structure.

\section{Numerical Simulation}

In this section, the proposed method is applied in a beam structure with different kinds of breathing crack. Numerical simulation is conducted to verify the effectiveness of the proposed damage indicator.

A cantilever beam with the geometrical properties (length $1000 \mathrm{~mm}$ and square cross-section $15 \mathrm{~mm} \times 15 \mathrm{~mm}$ ), and material properties (Young's modulus $210 \mathrm{GPa}$ and density $7800 \mathrm{~kg} / \mathrm{m}^{3}$ ) in Figure 1 was considered. The whole beam was divided into 10 elements. The approach proposed by Sinha et al. [9] is used to model crack for the finite element model of the beam. According to this stiffness matrix, the natural frequencies of the beam under different crack scenarios are calculated in Table 1. Five different crack scenarios are considered in this simulation. Intact scenario I is utilized to compare the natural frequencies with the other damage scenarios. It can be seen that natural frequencies change slightly with the increase of crack number and depth ratio, which means that it is difficult to detect the crack only from the natural frequencies.

The cantilever beam is excited by a sinusoidal input at its first natural frequency according to Table 1 . The breathing crack was assumed to be closed when the displacement of the nodes of the cracked element in y-direction is greater than zero for the applied excitation and to be open when that displacement

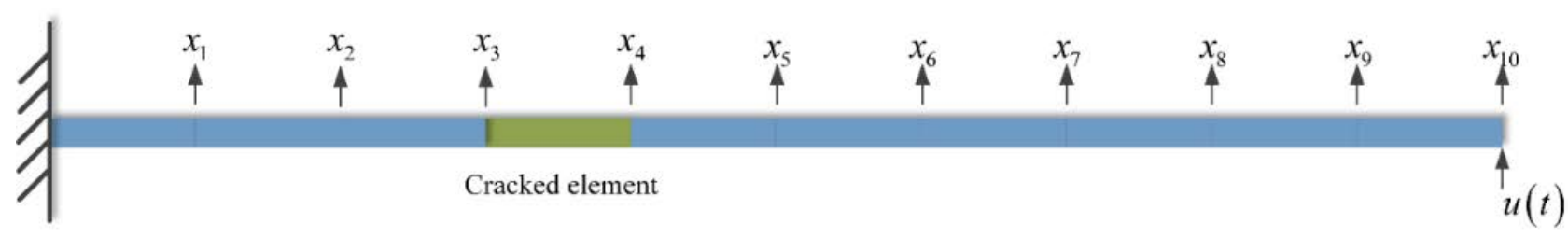

Figure 1. Finite element model of a cantilever beam. 
is less than zero. New mark-beta numerical method was used to calculate the response of the excited beam with $5000 \mathrm{~Hz}$ sampling frequency. Then, ODS of the crack beam can be got from the amplitude of the acceleration response at different nodes.

Figure 2 shows the ODSs of the cantilever beam for crack cases II and III. The two case scenarios have the same crack position at element 4 , but different crack depth ratio. It can be observed that the values of $2 x$ ODS change obviously from $5^{\text {th }}$ node, while the values of $1 x$ ODS is very smooth. That means the crack changes the value of higher harmonic ODS. Transmissibility of the ODSs are calculated further. The TODSs increase suddenly from the $5^{\text {th }}$ node in Figure 3, which shows the energy transmission changes from the $5^{\text {th }}$ node. Then, TEO is applied on the TODSs. As shown in Figure 4, the crossed dash line of the maximum values of the TTODSs indicate the crack position correctly. For crack cases II and III, the TTODSs almost overlap with each other, which shows that this indicators cannot distinguish different degrees of damage.

The same procedure is applied on the cases IV and V in Figures 5-7. The two crack locations are indicated correctly in Figure 7. But, for each one case, the amplitude values of the $4^{\text {th }}$ crack and $8^{\text {th }}$ crack with same crack depth ratio are different. The value near the boundary is greater than another. That might shows that the boundary influences the transmissibility through energy transfer.

Table 1. Crack scenarios and the corresponding natural frequency of the cantilever beam.

\begin{tabular}{ccccccc}
\hline \multirow{2}{*}{ Case scenarios } & \multicolumn{3}{c}{ Crack } & \multicolumn{4}{c}{ Frequency(Hz) } \\
\cline { 2 - 6 } & Size $(r)$ & Location (No.) & Mode 1 & Mode 2 & Mode 3 & Mode 4 \\
\hline I & No crack & No crack & 12.57 & 78.80 & 220.68 & 432.74 \\
II & 0.2 & 4 & 12.49 & 78.38 & 218.78 & 432.68 \\
III & 0.3 & 4 & 12.45 & 78.22 & 218.05 & 432.66 \\
IV & $0.2,0.2$ & 4,8 & 12.48 & 78.06 & 216.11 & 428.32 \\
V & $0.3,0.3$ & 4,8 & 12.45 & 77.76 & 214.33 & 426.64 \\
\hline
\end{tabular}

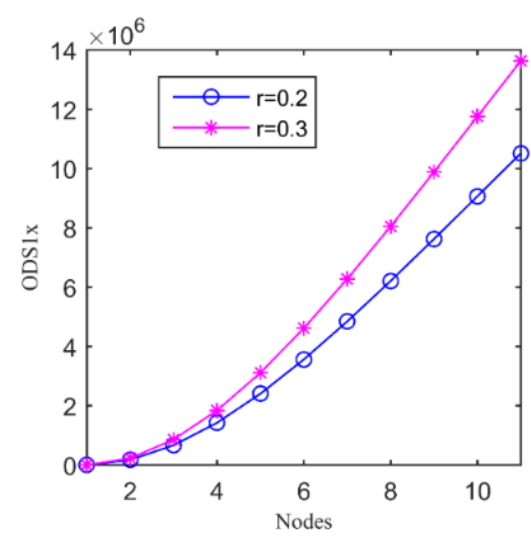

(a)

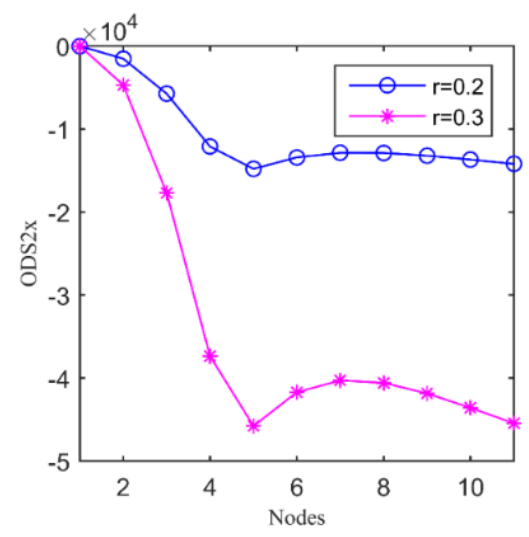

(b)

Figure 2. ODSs of case II and III: (a) 1x ODS; (b) 2x ODS. 


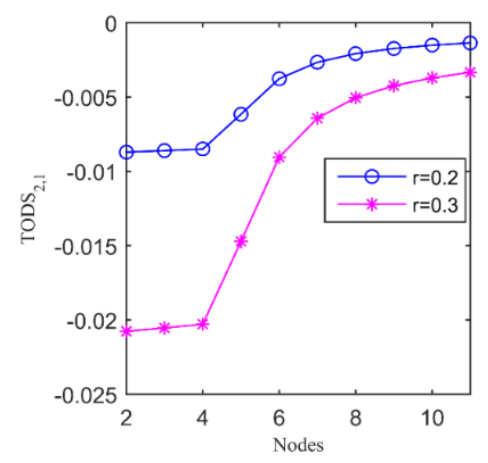

Figure 3. TODSs of case II and III.

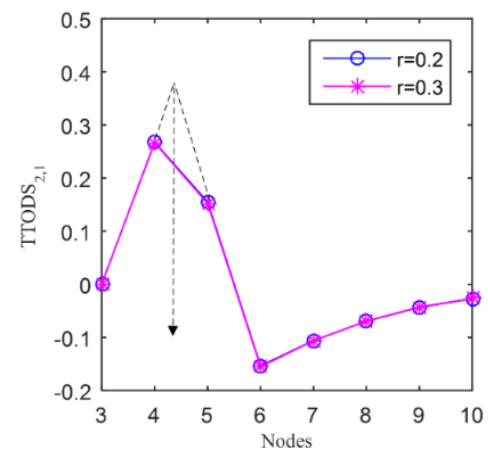

Figure 4. TTODSs of case II and III.

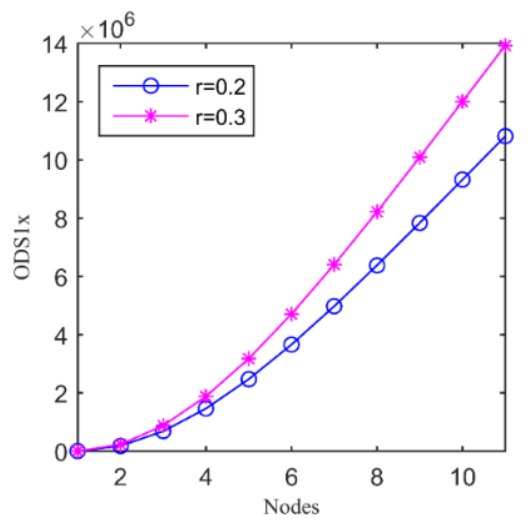

(a)

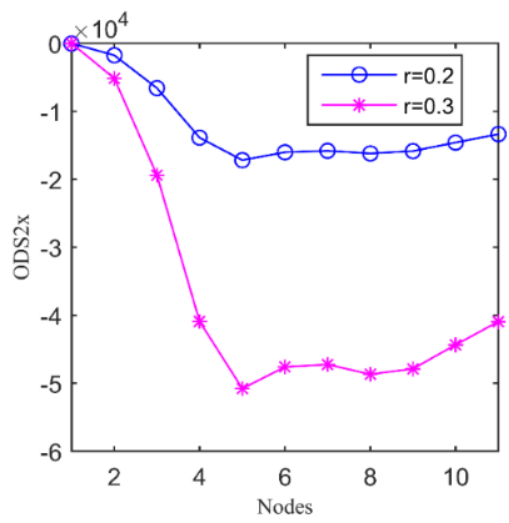

(b)

Figure 5. ODSs of case IV and V: (a) 1x ODS; (b) 2x ODS.

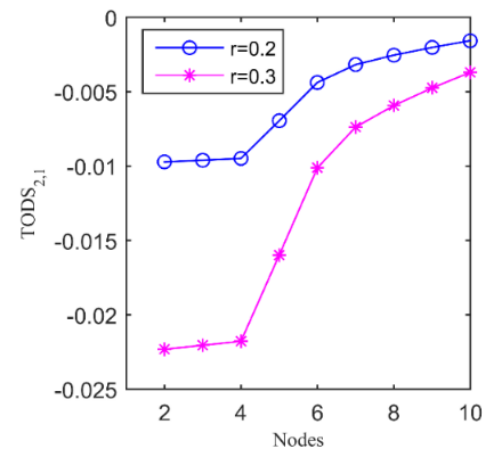

Figure 6. TODSs of case IV and V. 


\section{Experimental Verification}

The approach proposed in the preceding section is validated experimentally only using the response of beam structures under sine excitation in this section.

Cracked beam clamped at its right end is considered in this experiment. Figure 8 shows the test setup. Seven acceleration sensors are installed at equal interval of $6 \mathrm{~cm}$ along the beam from the fixed end. Three crack scenarios are studied in this experiment as shown in Table 2 . One single crack is created by saw cut between the $3^{\text {th }}$ and $4^{\text {th }}$ sensors with different depth. The corresponding natural frequencies are listed in Table 2.

After the experimental system is assembled, test is implemented follow on.

Step 1. Random excitation is generated to drive the shaker and vibration responses from all the seven acceleration sensors are recorded. The sampling frequency is $1024 \mathrm{~Hz}$.

Step 2. OMA method is used to calculate the natural frequencies from the time domain responses.

Step 3. Sinusoidal excitation with first natural frequency is generated to drive

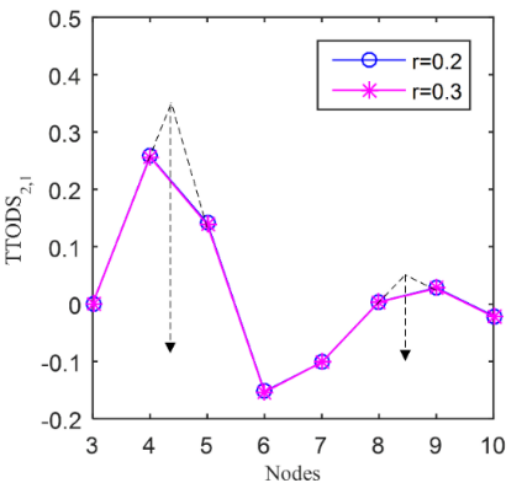

Figure 7. TTODSs of case IV and V.

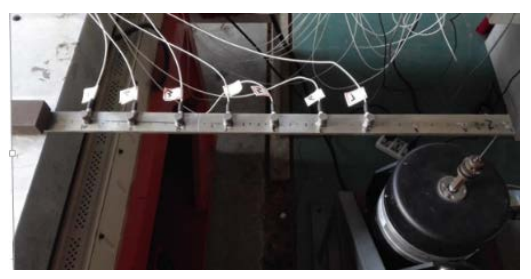

(a)

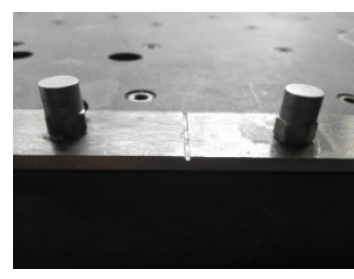

(b)

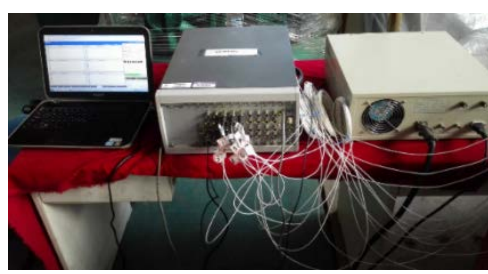

(c)

Figure 8. Test setup: (a) cantilever beam; (b) crack and acceleration sensor; (c) test equipment.

Table 2. Experimental crack scenarios and the corresponding natural frequencies of the beam.

\begin{tabular}{cccccc}
\hline \multirow{2}{*}{ Case scenarios } & \multicolumn{2}{c}{ Crack } & \multicolumn{3}{c}{ Frequency(Hz) } \\
\cline { 2 - 6 } & depth $(\mathrm{mm})$ & Location (Sensor No.) & Mode 1 & Mode 2 & Mode 3 \\
\hline I & 3.0 & $3 \sim 4$ & 50.50 & 153.00 & 323.75 \\
II & 1.5 & $3 \sim 4$ & 50.75 & 155.75 & 319.25 \\
III & 0.5 & $3 \sim 4$ & 50.50 & 157.25 & 322.50 \\
\hline
\end{tabular}


the shaker and responses from all the seven acceleration sensors are recorded. The sampling frequency is $512 \mathrm{~Hz}$.

Step 4 . The proposed method in Section 2 is used to identify the crack position with the responses.

The experimental data are processed using the proposed method for all the three crack scenarios. For crack scenario I, the crack location can be identified exactly from the TTODS in Figure 9(a). For crack scenario II, the negative peak of TTODS appears near the $3^{\text {th }}$ sensor in Figure 9 (b). For crack scenario III, the maximum peak of TTODS indicates that the crack is near the $4^{\text {th }}$ sensor in Figure 9 (c). The experimental results show that the crack can be detected by the proposed method, and the accuracy of the identified damage location falls with the decrease of crack depth.

Further research is carried out using the comparison with the modal curvature [10]. Modal curvature is a popular conventional damage detection method based on modal shape, and can be computed by the second-order central difference of the mode shape. Since the excitation frequency is the first natural frequency, ODS at $1 \mathrm{x}$ is the first modal shape. So, modal curvature can be applied here. All three crack scenarios are calculated using modal curvature as shown in Figure 10.

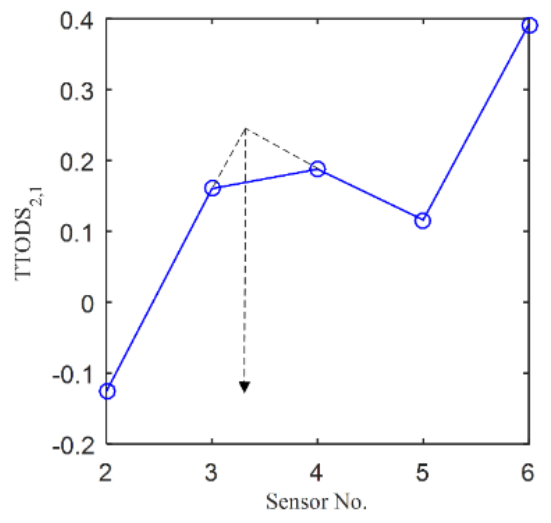

(a)

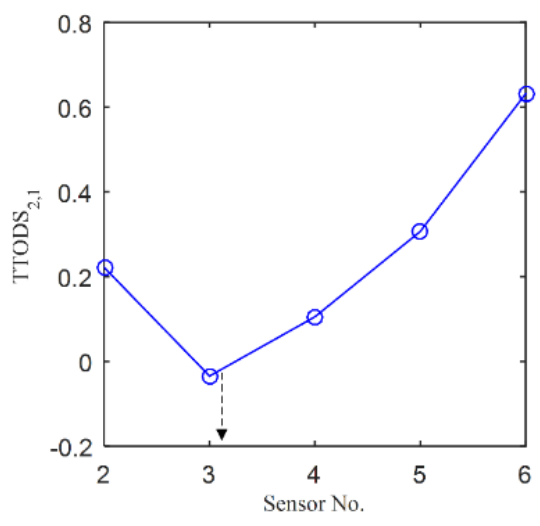

(b)

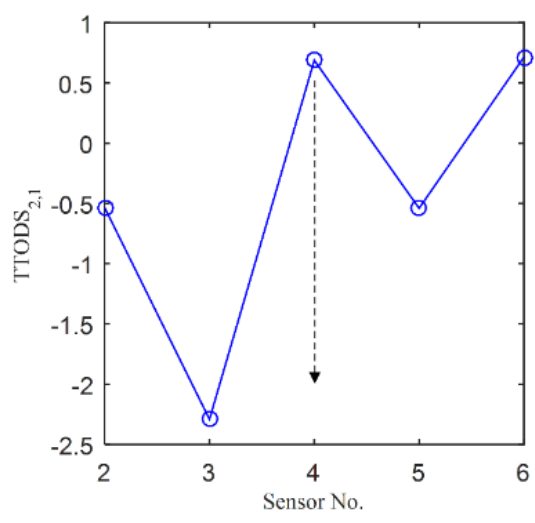

(c)

Figure 9. TTODSs of Crack detection for: (a) scenario I; (b) scenario II; (c) scenario III.

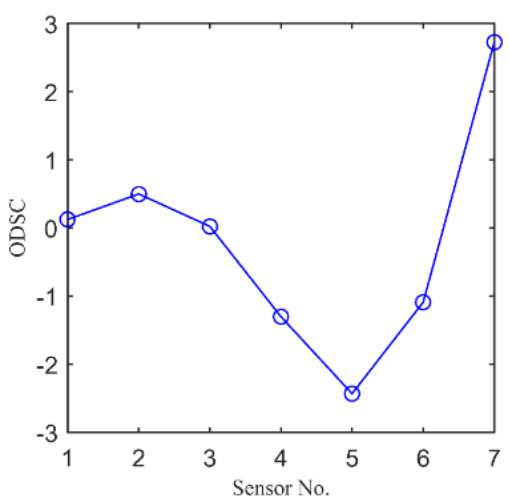

(a)

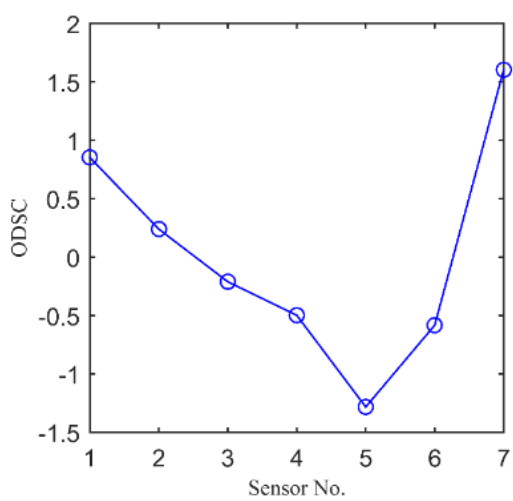

(b)

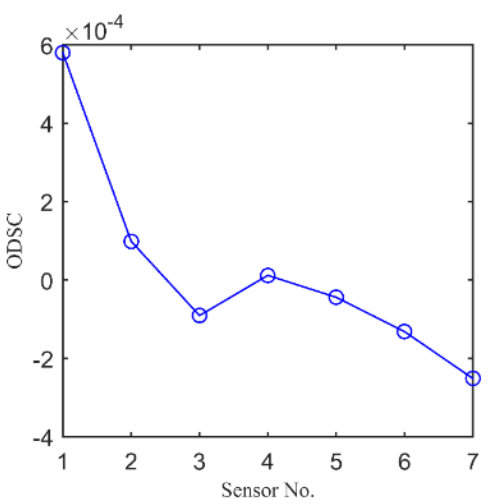

(c)

Figure 10. Crack detection using modal curvature for: (a) scenario I; (b) scenario II; (c) scenario III. 
It can be seen that the modal curvature can't locate the crack position at all. One reason might be there are not enough sensors. Another reason is the modal curvature is susceptible to noise. Thus, the proposed method using transmissibility of ODS has the capacity to locate the damage position.

\section{Conclusion}

Structure with breathing crack will generate higher harmonics of the frequency of excitation. Based on the properties of the ODS and transmissibility, a new damage indicator is proposed in this paper. The transmissibility of higher harmonic ODS and 1x ODS is developed to show the energy transfer under the influence of crack. Since the higher harmonics are induced by crack breathing, the transmissibility changes near the crack location observed from the numerical simulation. TEO is introduced to reveal the singularity further. The results show that the proposed indicator can identify the location of crack accurately both in the case with single crack and two cracks.

\section{Acknowledgements}

This work was supported by China Postdoctoral Science Foundation (2018M633633XB), and National Natural Science Foundation of China (Grant No. 11802279).

\section{Conflicts of Interest}

The authors declare no conflicts of interest regarding the publication of this paper.

\section{References}

[1] Fan, W. and Qiao, P. (2011) Vibration-Based Damage Identification Methods: A Review and Comparative Study. Structural Health Monitoring, 9, 83-111. https://doi.org/10.1177/1475921710365419

[2] Ratcliffe, C.P. (2000) A Frequency and Curvature Based Experimental Method for Locating Damage in Structures. Journal of Vibration and Acoustics, 122, 324-329. https://doi.org/10.1115/1.1303121

[3] Yoon, M.K., Heider, D., Gillespie, J.W., Ratcliffe, C.P. and Crane, R.M. (2010) Local Damage Detection with the Global Fitting Method using Operating Deflection Shape Data. Journal of Nondestructive Evaluation, 29, 25-37. https://doi.org/10.1007/s10921-010-0062-8

[4] Zhang, Y., Lie, S.T. and Xiang, Z. (2013) Damage Detection Method Based on Operating Deflection Shape Curvature Extracted from Dynamic Response of a Passing vehicle. Mechanical Systems and Signal Processing, 35, 238-254. https://doi.org/10.1016/j.ymssp.2012.10.002

[5] Feng, K. and Li, Z. (2016) A Novel Superposed Waveform Method for Damage Detection of Composite Laminates. Nondestructive Testing and Evaluation, 31, 61-76. https://doi.org/10.1080/10589759.2015.1057586

[6] Asnaashari, E. and Sinha, J.K. (2014) Development of Residual Operational Deflection Shape for Crack Detection in Structures. Mechanical Systems and Signal 
Processing, 43, 113-123. https://doi.org/10.1016/j.ymssp.2013.10.003

[7] Zhang, H., Schulz, M., Naser, A., Ferguson, F. and Pai, P. (1999) Structural Health Monitoring using Transmittance Functions. Mechanical Systems and Signal Processing, 13, 765-787. https://doi.org/10.1006/mssp.1999.1228

[8] Mottershead, J. (1998) On the Zeros of Structural Frequency Response Functions and Their Sensitivities. Mechanical Systems and Signal Processing, 12, 591-597. https://doi.org/10.1006/mssp.1998.0167

[9] Sinha, J., Friswell, M. and Edwards, S. (2002) Simplified Models for the Location of Cracks in Beam Structures using Measured Vibration Data. Journal of Sound and Vibration, 251, 13-38. https://doi.org/10.1006/jsvi.2001.3978

[10] Pandey, A.K., Biswas, M. and Samman, M.M. (1991) Damage Detection from Changes in Curvature Mode Shapes. Journal of Sound and Vibration, 145, 321-332. https://doi.org/10.1016/0022-460X(91)90595-B 